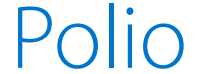

\section{What is polio?}

Poliomyelitis (or 'polio') is a viral infection that can cause paralysis and death. In the past, polio was common, especially in children. Now, due to immunisation, polio is rare in most parts of the world, although it persists in some areas. The last reported case of wild (disease-causing) polio in Australia occurred in 1978.

\section{What are the symptoms?}

- The majority of people infected with polio do not have symptoms.

- A minor illness causing fever, headache, lethargy, nausea and vomiting occurs in approximately 10 per cent of people who are infected with the virus. Most of these people completely recover. However, approximately 2 per cent go on to experience severe muscle pain with back or neck stiffness, called non-paralytic aseptic meningitis (inflammation of the lining of the brain without weakness).

- Less than 1 per cent of people who are infected develop severe weakness called acute flaccid paralysis. This usually affects the limbs but it can also affect the muscles of the head, neck and diaphragm muscle, which is used for breathing. Most people with acute flaccid paralysis recover, although the recovery is not complete in all people and some die.

\section{How is it spread?}

- Polio is a highly infectious disease spread by close contact with an infected person, via contact with minute amounts of faeces (eg, on unwashed hands) or from droplets from the throat of an infected person.

- Untreated sewerage that comes into contact with foods or drinking water can spread polio in parts of the world where there is poor sanitation.

- The polio virus enters the body through the nose or mouth and infection starts in the gut. It then enters the blood stream and is carried to other parts of the body, including the nervous system.

- The time from being exposed to the polio virus and getting sick can range from 3 to 35 days, but is commonly 7 to 14 days.

- Cases are most infectious from 10 days before onset of symptoms to 10 days after the onset of symptoms.

- People can continue to shed the virus in their faeces for up to six weeks.

\section{Who is at risk?}

- Owing to immunisation, Australia is currently free from polio.

- The World Health Organization is currently working towards eradicating polio worldwide but in 2007 it still exists in parts of Africa and South Asia.
- People who are not immune may become infected in countries where polio still exists. They may then bring the infection with them when they travel to another country.

\section{How is it prevented?}

Immunisation protects people against polio. While transmission does not currently occur in Australia, the polio virus could be imported and spread among unimmunised populations.

- For all children, a course of three injections at two, four and six months of age, with boosters at 4 years of age, is recommended.

- For some adults (travellers to countries with polio and health care workers who may look after patients with polio), a booster is recommended every 10 years.

- Adequate treatment of sewerage and provision of safe drinking water and foods is also important to prevent the disease from spreading.

\section{How is it diagnosed?}

- The doctor may suspect polio based on the person's symptoms and signs. However, some other infections can cause similar symptoms. Acute flaccid paralysis can also have other causes and these people need blood tests and stool tests to test if their symptoms are caused by polio virus. A national polio expert committee decides if the symptoms and tests could be polio.

- Isolation of the virus from stools, throat or spinal cord fluid is required to confirm the diagnosis of polio.

- Special studies are needed to distinguish the vaccine strain of the virus from the wild (disease-causing) virus.

\section{How is it treated?}

There is no cure for polio and treatment is mainly to support cases with acute flaccid paralysis while their weakness is severe. Cases with acute flaccid paralysis may require intensive care to assist breathing.

\section{What is the public health response?}

- Hospitals, laboratories, school principals and childcare centres must notify suspected cases of polio including acute flaccid paralysis to the local public health unit.

- Public health units will investigate suspect cases and review possible sources of infection to prevent further spread.

- If a case is detected in Australia, people who are at risk may need to be immunised again against polio.

For more information, please contact your doctor, local public health unit or community health centre.

NSW 